\title{
Capsaicin may slow PSA doubling time: case report and literature review
}

\author{
Bojana Jankovic, BSc, MSc, MD; ${ }^{*} D$. Andrew Loblaw, BSc, MD, MSc, FRCPC, CIP; ${ }^{\dagger}$ \\ Robert Nam, MD, MSc, FRCSC ${ }^{\ddagger}$
}

\begin{abstract}
Capsaicin is the main pungent component of chili peppers. This is the first case, to our knowledge, that describes prostatespecific antigen (PSA) stabilization in a patient with prostate cancer, who had biochemical failure after radiation therapy. A 66year-old male underwent radiotherapy treatment for a T2b, Gleason $7(3+4)$ adenocarcinoma of the prostate, with a PSA level of $13.3 \mathrm{ng} / \mathrm{mL}$ in April 2001. He had 3-dimensional conformal radiotherapy of 46 Gy in 23 fractions to the prostate and pelvis, and a prostate boost of 30 Gy in 15 fractions. Radiotherapy was completed in May 2001 and PSA nadired in January 2002 (0.57). Due to the continued PSA rise, the patient was started on bicalutamide (50 mg orally, daily) and leuprolide acetate (1 dose of $22.5 \mathrm{mg}$ intramuscularly) in July 2005 when PSA was $38.5 \mathrm{ng} / \mathrm{mL}$. Due to poor tolerance of androgen ablation therapy, the patient discontinued treatment and started taking $2.5 \mathrm{~mL}$ of habaneros chili sauce, containing capsaicin, 1 to 2 times a week in April 2006. Prostate-specific antigen doubling time (PSAdt) increased from 4 weeks before capsaicin to 7.3 months by October 2006. From October 2006 until November 2007, the patient remained on capsaicin (2.5 to $15 \mathrm{~mL}$ daily) and his PSA was stable (between 11 to $14 \mathrm{ng} / \mathrm{mL}$ ). By January 2008, his PSA rose to 22.3 and he has maintained a PSAdt between 4 and 5 months, where it presently remains. Due to the patient's continued PSA rise, he was restarted on bicalutamide (12.5 mg daily). Apart from PSA relapse, the patient remains free of signs or symptoms of recurrence.
\end{abstract}

Can Urol Assoc J 2010;4(1):E9-E11

\section{Introduction}

In vitro studies of capsaicin ( $\mathrm{N}$-vanillyl-8-methyl-1-nonenamide), the main pungent component of red pepper, have demonstrated its anti-proliferative and apoptotic effect in various cancer cell lines. To our knowledge, no human trials have been reported on its efficacy in the clinical setting. We report a case of a patient with recurrent prostate cancer, who experienced a slowing of his prostate-specific antigen doubling time (PSAdt) while taking capsaicin.

\section{Case report}

A 66-year-old man presented in April 1999 with a palpable prostate nodule and a prostate-specific antigen (PSA) level of $9 \mathrm{ng} / \mathrm{mL}$. He was found to have a T2a and Gleason score $7(3+4)$ adenocarcinoma of the prostate involving $70 \%$ of the surface area of his core biopsies. Due to coronary artery bypass grafting for coronary artery disease and a total knee replacement, his radiotherapy was delayed to April 2001. On examination in February 2001, his clinical stage was T2b and PSA had risen to $13.3 \mathrm{ng} / \mathrm{mL}$. He was treated with 3-dimensional conformal radiotherapy of 46 Gy in 23 fractions to the pelvis and prostate with a prostate boost of an additional 30 Gy in 15 fractions (total prostate dose 76 Gy in 38 fractions) from April 3 to May 29, 2001. No adjuvant hormonal therapy was administered. At the time of his radiation, his medications were lisinopril, furosemide, celecoxib, docusate sodium, acetylsalicylic acid and acetaminophen/codeine, as needed. By January 2002 , his PSA nadired at $0.57 \mathrm{ng} / \mathrm{mL}$, but rose to $7.6 \mathrm{ng} / \mathrm{mL}$ by November 2004 (PSAdt = 12 months); by July 2005, his PSA had risen to $38.5 \mathrm{ng} / \mathrm{mL}$ (PSAdt $=3.6$ months) (Fig. 1).

The patient was started on bicalutamide $50 \mathrm{mg}$ orally, daily and had a leuprolide acetate $22.5 \mathrm{mg}$ intramuscular injection in July 2005. His other medications remained the same. After 2 doses of bicalutamide, the patient discontinued the bicalutamide due to adverse reactions including lightheadedness, hallucinations and pre-syncope, events which caused him to present to the emergency room. He had an excellent response on leuprolide (1 injection), with his PSA decreasing from $38.52 \mathrm{ng} / \mathrm{mL}$ in July to $0.25 \mathrm{ng} / \mathrm{mL}$ in December 2005. He discontinued leuprolide due to side effects, including significant proximal muscle weakness, decreased tone diffusely, gynecomastia and hot flashes not relieved by venlafaxine. The patient's PSA again started to rise with a doubling time of 4 weeks ( 3 measured values) from January (PSA $1.4 \mathrm{ng} / \mathrm{mL}$; testosterone $[\mathrm{T}]=7.0 \mathrm{nmol} / \mathrm{L}$ ) to April 2006 (PSA $7.3 \mathrm{ng} / \mathrm{mL} ; \mathrm{T}=9.6 \mathrm{nmol} / \mathrm{L}$ ).

The patient started taking $2 \mathrm{~mL}$ of habaneros chili sauce, containing capsaicin, 1 to 2 times per week in April 2006. The capsaicin concentration in the habanernos sauce was 


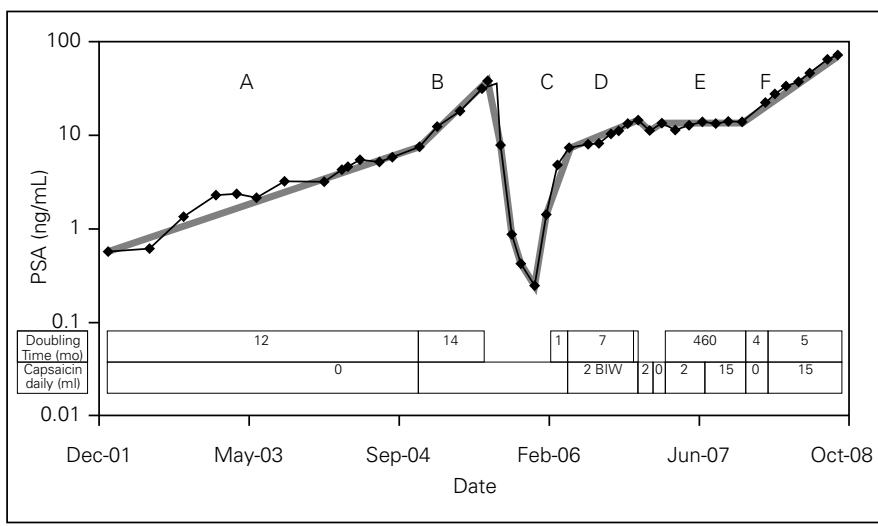

Fig. 1. Prostate-specific antigen (PSA) values plotted over time. PSA doubling time and capsaicin consumption are overlaid. Slope $A$ represents a slowly rising PSA post-radiotherapy. Slope B represents acceleration of the PSA kinetics. Slope $C$ is a fast PSA doubling time during eutestosteronemia after the cessation of androgen deprivation therapy. Slope D is a slowed PSA doubling time after occasional use of capsaicin. Slope E shows stabilization of PSA with regular use of capsaicin. Slope F shows PSA progression despite regular use of capsaicin.

calculated to be $454 \mathrm{ug} / \mathrm{mL}$. Based on the next 5 PSA values, the doubling time decreased to 7.3 months, calculated in October 2006.

From the end of October 2006, when PSA was $13.26 \mathrm{ng} / \mathrm{mL}$, the patient started taking $2 \mathrm{~mL}$ of the sauce daily. By January 2007, the PSA decreased to $11.3 \mathrm{ng} / \mathrm{mL}$. From the end of January 2007 to the end of February 2007, he stopped taking the habaneros sauce, and his PSA rose to $13.57 \mathrm{ng} / \mathrm{mL}$ in mid-February. After restarting the sauce at $2 \mathrm{~mL}$ per day in March 2007 and increasing it to $15 \mathrm{~mL}$ per day in July 2007, the PSA remained stable until November 2007.

The patient stopped taking capsaicin in November 2007 and his PSA increased to $22.3 \mathrm{ng} / \mathrm{mL}$ by January 2008 . By April 2008, he was on capsaicin again, $15 \mathrm{~mL}$ daily. The patient developed congestive heart failure, and was started on carvedilol in November 2007, which coincided with a rise in PSA velocity (doubling time 3.5 months). In February 2008, he took half of the carvedilol dose and his doubling time increased to 5 months. In July 2008, it was suggested that the patient start taking 1000 IU of vitamin D daily. No change in PSAdt was observed. His last PSA was $72.19 \mathrm{ng} / \mathrm{mL}$ (September 2008), and has been increasing at a doubling time of 4 to 5 months since February 2008. At the time of submission, he has been restarted on bicalutamide $12.5 \mathrm{mg}$ orally, daily due to poor tolerance previously.

Other than what is described above, the patient stated that he did not change his diet in any meaningful way or take other natural supplements from October 2006 to the time of submission of this paper.

Apart from biochemical failure, the patient has had no signs or symptoms of local or distant recurrence. In January 2003, he had a transrectal ultrasound-guided biopsy, which was negative for malignancy. His last staging investiga- tions, including computed tomography scan of thorax, abdomen and pelvis and a bone scan done in February 2008, were negative. At no time did the patient report any side effects from the capsaicin, including any gastrointestinal upset or discomfort.

\section{Discussion}

Capsaicin has been shown to inhibit growth and cause apoptosis of prostate cancer cell lines in vitro and in vivo.

In vitro experiments in androgen receptor (AR) positive prostate cancer cells (LNCaP) show that the administration of capsaicin inhibits growth and causes apoptosis in 93\% of the cells. It also results in a downregulation of AR and PSA expression in the presence of dihydrotestosterone, and increase in p53, p21 and Bax proteins which are involved in cell cycle regulation and apoptosis. ${ }^{1}$

Capsaicin also has anti-proliferative activity in androgen-independent (AI) and AR negative cells and PC-3; it was found to induce apoptosis in $75 \%$ of the treated cells. ${ }^{1}$ The mechanism for this activity likely involves multiple pathways involved in cell growth and stress-response. In Al cells, capsaicin inhibited translocation of transcription factor, NF- $\kappa B$, to the nucleus where it acts as a transcription factor for cytokines, cell cycle regulatory proteins and anti-apoptotic proteins. ${ }^{1}$ Capsaicin was also shown to induce apoptosis via reactive oxygen species (ROS) formation, dissipation of mitochondrial inner transmembrane potential and caspase 3 activation in PC-3 cells. ${ }^{2}$ Further studies have shown that ROS generation may be necessary for ceramide accumulation and cell growth inhibition by capsaicin. ${ }^{3}$ Pathways involving Jun N-terminal Kinase (JNK) and extracellular signal-regulated kinase (ERK) cascades have also been implicated in anti-proliferative effects of capsaicin. ${ }^{3}$ New data demonstrates that capsaicin is associated with upregulation of endoplasmic reticulum stress-regulated gene GADD153/CHOP in PC-3 cells. Blockage of expression of this gene significantly reduced capsaicin-induced cell death. ${ }^{4}$

Capsaicin has shown activity in other cancer cell lines. It induced apoptosis of glioma cells, ${ }^{5}$ hepatocellular carcinoma cells, ${ }^{6}$ human esophagus epidermoid cells, ${ }^{7}$ human leukemia cells ${ }^{8}$ and human breast cancer cells. ${ }^{9}$ Activation of caspase3 was involved in capsaicin-induced cell death. . $^{-9}$

In vivo experiments support the results seen in cell lines. Capsaicin significantly slowed tumour growth in androgenindependent prostate cancer (PC-3) xenografts in mice. Untreated mice had, on average, 4.5 times larger tumour volume than those receiving capsaicin starting on the first day of PC-3 inoculation $(p=0.017) .{ }^{1}$ Tumour growth of the PC-3 cells was reduced by $40 \%$ when capsaicin was administered to mice 4 weeks after tumour inoculation. ${ }^{2}$

We report PSA stabilization on capsaicin in a patient with PSA relapse after radiotherapy for a T2b, Gleason score 
7 adenocarcinoma of the prostate. At biochemical failure, the patient did not tolerate androgen ablation therapy and started taking habanero sauce containing capsaicin 2.5 to $5 \mathrm{~mL}$ daily in April 2006.

During the periods the patient was on capsaicin, his PSAdt lengthened or PSA stabilized. Prostate-specific doubling time trends in untreated, relapsed prostate cancer patients are poorly documented. While one would expect a dramatic change in PSAdt as the patient recovers testosterone function, and a gradual lengthening of PSAdt over time in some patients, we observed a notable change in PSAdt even after his testosterone normalized before and after starting capsaicin. When the patient stopped capsaicin, a PSA rise or decrease in doubling time was consistently observed. We are unaware of other changes in the patient's diet, supplementation or medication that was associated with these changes. There is evidence from in vitro and in vivo studies that capsaicin has anti-proliferative and apoptotic activity in prostate cancer and other cell lines.

\section{Conclusion}

To our knowledge, this is the first case of observed delay in PSA progression in a prostate cancer patient that has been associated with use of capsaicin. While we find this observation interesting, a cause-effect relationship cannot be concluded and should be considered hypothesis generating.

Given that capsaicin is inexpensive, easy to administer, has no known side effects and may have tumouristatic properties (at least based on in vitro work), it holds promise as an adjunctive treatment option. It may be useful in prevention, in slowing the growth of prostate cancer in patients on active surveillance, or in patients who have recurrent disease before the next line of definitive therapy. However, we believe that clinical trials are needed to evaluate the role of capsaicin in patients with prostate cancer before capsaicin is widely adopted. Further studies are also needed to further elucidate the mechanisms of capsaicin-mediated inhibition of cell growth and apoptosis.

From the ${ }^{*}$ Faculty of Medicine, UBC, Vancouver, BC; ${ }^{\dagger}$ Department of Radiation Oncology, Odette

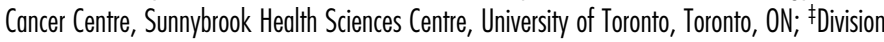
of Urology, Sunnybrook Health Sciences Centre, Toronto, ON

Competing interests: Dr. Loblaw declares that he co-held a patent pending on a supplement that contained capsaicin.

This paper has been peer-reviewed.

\section{References}

1. Mori A, Lehmann S, O'Kelly J, et al. Capsaicin, a component of red peppers, inhibits the growth of androgen-independent, p53 mutant prostate cancer cells. Cancer Res 2006; 66:3222-9.

2. Sanchez AM, Sanchez MG, Malagarie-Cazenave $S$, et al. Induction of apoptosis in prostate tumor pc-3 cells and inhibition of xenograft prostate tumor growth by the vanilloid capsaicin. Apoptosis 2006; 11:89-99.

3. Sanchez AM, Malagarie-Cazenave S, Olea N, et al. Apoptosis induced by capsaicin in prostate pc-3 cells involves ceramide accumulation, neutral sphingomyelinase, and jnk activation. Apoptosis 2007;12:2019-24.

4. Sanchez AM, Martínez-Botas J, Malagarie-Cazenave S, et al. Induction of the endoplasmic reticulum stress protein gadd153/chop by capsaicin in prostate pc-3 cells: A microarray study. Biochem Biophys Res Commun 2008;372:785-91.

5. Qiao $S$, Li W, Tsubouchi $R$, et al. Involvement of peroxynitrite in capsaicin-induced apoptosis of c6 glioma cells. Neurosci Res 2005;51:175-83.

6. Jung MY, Kang HJ, Moon A. Capsaicin-induced apoptosis in sk-hep-1 hepatocarcinoma cells involves bcl2 downregulation and caspase-3 activation. Cancer Lett 2001;165:139-45.

7. Wu CC, Lin JP, Yang JS, et al. Capsaicin induced cell cycle arrest and apoptosis in human esophagus epidermoid carcinoma ce 81t/vgh cells through the elevation of intracellular reactive oxygen species and ca2+ productions and caspase-3 activation. Mutat Res 2006;601:71-82.

8. Tsou MF, Lu HF, Chen SC, et al. Involvement of bax, bcl-2, ca2+ and caspase-3 in capsaicin-induced apoptosis of human leukemia hl-60 cells. Anticancer Res 2006;26:1965-71.

9. Tuoya , Baba N, Shimoishi Y, et al. Apoptosis induction by dohevanil, a dha substitutive anaolog of capsaicin, in mcf-7 cells. Life Sci 2006; 78:515-9.

Correspondence: Dr. Andrew Loblaw, Department of Radiation Oncology, Odette Cancer Centre, Sunnybrook Health Sciences Centre, University of Toronto, 2075 Bayview Ave., Toronto, ON M4N 3M5; fax: 416-480-6002; andrew.loblaw@sunnybrook.ca 